\title{
IMCA: An Efficient In-memory Convolution Accelerator
}

\author{
Hasan Erdem Yantır, Ahmed M. Eltawil, Senior Member, IEEE, and Khaled N. Salama, Senior Member, IEEE
}

\begin{abstract}
Traditional convolutional neural network (CNN) architectures suffer from two bottlenecks; computational complexity and memory access cost. In this study, an efficient in-memory convolution accelerator (IMCA) is proposed based on associative in-memory processing to alleviate these two problems directly. In the IMCA, the convolution operations are directly performed inside the memory as in-place operations. The proposed memory computational structure allows for a significant improvement in computational metrics, namely, TOPS/W. Furthermore, due to its unconventional computation style, the IMCA can take advantage of many potential opportunities such as constant multiplication, bit-level sparsity, and dynamic approximate computing, which, while supported by traditional architectures, require extra overhead to exploit, thus reducing any potential gains. The proposed accelerator architecture exhibits a significant efficiency in terms of area and performance, achieving around 0.65 GOPS and 1.64 TOPS/W at 16-bit fixed-point precision with an area less than $0.25 \mathrm{~mm}^{2}$.
\end{abstract}

Index Terms-Artificial intelligence, convolutional neural networks, in-memory computing, associative processors, non-von Neumann architectures, convolution accelerator

\section{INTRODUCTION}

$\mathbf{T}$ HE human brain is the most efficient processor known until now; therefore, researchers want to mimic the brain in performing computations for tasks that mimic human intelligence such as reasoning, planning, learning, etc. Collectively, these tasks are studied in the field of Artificial Intelligence (AI), where computations are performed using brain-inspired approaches. An adult brain has an average of 8.6 billion neurons, and 500 trillion synapses [1]. Considering a case-inpoint where a study to simulate one second of the human brain activity requires 82,944 processors running for 40 minutes and dissipating 9.89 MW in total [2]. This is especially intriguing since the real human brain consumes approximately around 10-20 W [3]. Even though AI systems achieve great success in performing complex intelligence tasks, this achievement comes with an overwhelming cost. The existing architectures for AI focus on utilizing massive parallelism to address the computational needs; however, the issue of memory access is still largely not addressed. This fact forces researchers to find a much more efficient representation of the AI systems

Manuscript received August 09, 2020; revised November 28, 2020; accepted December 14, 2020. (Corresponding author: Hasan Erdem Yantır.)

Hasan Erdem Yantır and Khaled N. Salama are with the Sensors Lab, Advanced Membranes \& Porous Materials Center (AMPMC), Computer, Electrical and Mathematical Sciences and Engineering Division, King Abdullah University of Science and Technology (KAUST), Thuwal, 23955-6900, Saudi Arabia; (e-mail: \{hasan.yantir, khaled.salama\} @kaust.edu.sa)

Ahmed M. Eltawil is with the Computer, Electrical and Mathematical Sciences and Engineering Division, King Abdullah University of Science and Technology (KAUST), Thuwal 23955-6900, Saudi Arabia; (e-mail: \{ahmed.eltawil\}@kaust.edu.sa) in the computation domain as well as efficient processing architectures [4]. This study aims to address these challenging issues, namely, computational complexity and off-chip memory access in traditional AI architectures specifically for convolution neural networks (CNN).

One successful specific type of AI systems is CNNs, which can outperform humans in many visual perception tasks [5]. As an example case, the preliminary results show that $\mathrm{CNN}$-based AI systems can outperform pathologists in diagnosing some cancer types both in terms of throughput and accuracy [6]. Regarding these promising applications and their outcomes, it is highly expected that the demand for AI is projected to increase tremendously to span every major industry from medicine to security. On the other hand, modern CNN architectures require hundreds of convolution layers stacked to each other, performing billions of operations for specific input data. Furthermore, AI systems need more capable hardware as the complexity of the required tasks increases (from classification to segmentation, detection, and localization). From the implementation perspective, shrinking in the transistor dimensions has approached its limits [7], so it is difficult to shrink the transistor dimensions further to obtain more performance. This reality makes the efficient implementation of AI-based systems on traditional processors limited. In order to meet the unique needs of AI-based systems, various architectures were proposed as possible contenders such as GPUs [8], or application-specific processors like Google TPU [9], and Intel Movidius [10] to accelerate AI tasks. These architectures focus on utilizing massive parallelism to address the computational needs; however, the issue of memory access is still largely not addressed. As the CNN complexity increases, the architecture becomes more memory dominant since the total number of weights and operations increases proportionally, leading to an increase in data access and an associated increase in energy consumption, ultimately limiting performance. As an example case from [11], reading two 32-bit numbers from $8 \mathrm{~KB}$, onchip SRAM consumes 100x more energy than performing 32-bit addition on these numbers in $45 \mathrm{~nm}$ technology. The memory bottleneck is also an issue for more advanced technologies (e.g., FinFET) since most of the energy is spent on communication. The ratio becomes much higher when the data access is from DRAM. Considering the enormous amount of calculations required on huge amounts of data for AI tasks, these conventional accelerator architectures are still challenged by the memory bottleneck problem. This is caused due to the processor-centric design principles applied since the prevalence of von Neumann architectures. However, current requirements of AI computation needs memory-centric design principles to address the memory bottleneck. 
In summary, this study proposes an In-Memory Convolution Accelerator (IMCA) for CNN-based AI systems. The IMCA combines processing and storage in the same location by following the principles of associative computing [12]. Processing on the data is performed without moving the data, i.e., truly in-place. This methodology seems very plausible since the brain similarly performs both processing and storage in the same place and at the same time. Additionally, the proposed architecture maximizes the computational density by exploiting memory rows as simple processors.

The rest of the paper is organized as follows: In the following section, the background information on CNNs and associative in-memory computing are presented together with related works. Section III introduces the proposed accelerator architecture in detail, together with architectural novelties and capabilities. Experimentation and evaluation results are discussed in Section IV. The final section concludes the work.

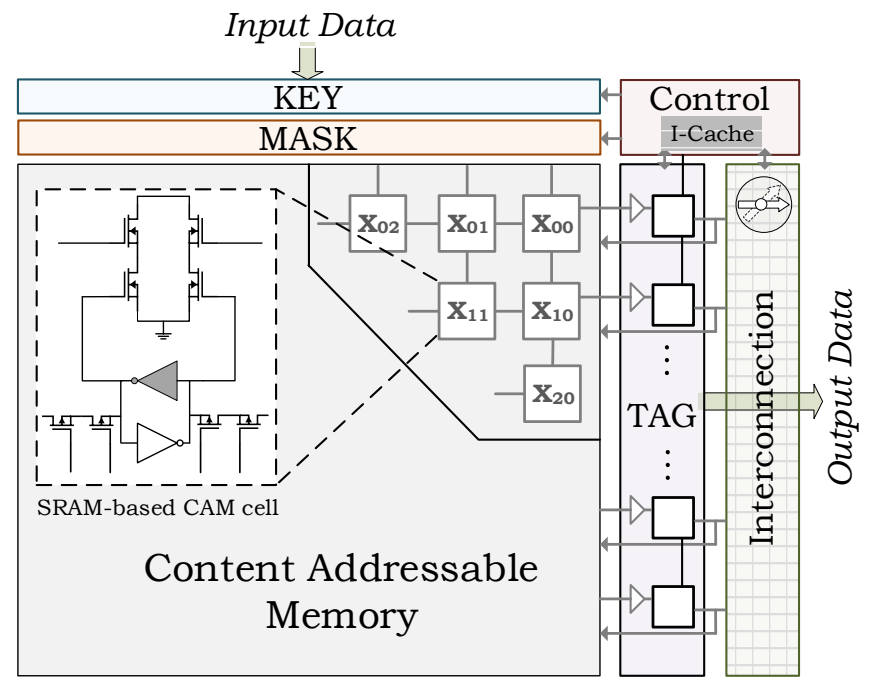

Fig. 1: Associative processor architecture

\section{BACKGROUND}

\section{A. Associative Processors}

The research on in-memory computing aims to cope with the barrier of memory bottleneck by processing the data inside the memory without moving it between the memory and the processor. The paradigm proposes replacing the logic with memory structures to virtually eliminate the need for memory load/store operations during computation. At this point, Associative Processing (AP) [12] seems as a very promising computational paradigm. The overall architecture of an associative processor is presented in Figure 1, which consists of content addressable memory (CAM), controller, and interconnection. To perform the operations in the memory, APs exploit the associativity feature of CAMs. CAM keeps the data on which operations are performed. CAM has some special registers to manage the data, such as key, mask, and tag. These registers are used to search specific content in the selected (masked) columns of the CAM. The key register stores the value that is compared against or written to the CAM. Mask register indicates the columns that are activated during comparison or write. The rows matched by a compare operation are marked in the tag register. The rows tagged with logic-1 means that the corresponding CAM row has been matched with the given key and mask value. For example, if we use key 010 and mask 110 to the CAM, the tag bits of the corresponding rows whose third and second bits are logic- 0 and logic-1 respectively become logic- 1 . The data movement to the CAM can be performed in two ways; column-parallel, row-serial, or column-serial, row-parallel. For the first way, the data that need to be placed inside a CAM row are stored in the key register. When the corresponding row's tag register becomes logic-1 together with the write enable signal, the key data is written to the corresponding row. This method is used while moving the data inside the CAM from the sensor or main memory. In the column-serial, row-parallel method, a single column of the CAM array can be written at once. This communication is used while transferring the data columnwise between the APs. In the same manner, the CAM cells can be read column-wise by using the tag register. Furthermore, it can be read in the same way for SRAM memories.

In traditional processor architectures, operands are read from memory and transferred to the processor. On the contrary, AP performs the required operations over data via consecutive compare and write operations on CAM. During these operations, the corresponding look-up table (LUT) of the performed operation is referenced [12]. In order to visualize the associative computing more comprehensively, Figure 2 illustrates the complete flow for in-place addition of two 4element, 2-bit signed vectors stored in a memory, A and B, i.e., $\mathrm{B}[\mathrm{i}]=\mathrm{B}[\mathrm{i}]+\mathrm{A}[\mathrm{i}], \mathrm{i}=0 \ldots 3$. The tables in the first row correspond to the LUT for addition required for the compare and write, and the others show the change in the memory (i.e., CAM) content together with the key/mask values and the tag status. The LUT entries show the required data for compare and write operations (if the compare matches) in order. At the start, A contains (i.e., columns $1-0)$ the values of $[1 ;-2 ; 1$; -1] and B (i.e., columns 3-2) contains the values of [1; -2; 0 ; -2] in binary two's complement. Cr (carry) column (i.e., column 4) is all 0s. The highlighted LUT entry points to the applied key on the masked columns. Each entry corresponds to a combination of $\mathrm{Cr}, \mathrm{B}, \mathrm{A}$ bits. Even though there are eight $\left(2^{3}\right)$ combinations, only four are used since others do not affect the operation. In each memory of the figure, the key value from the LUT's compare column is searched in the masked columns of the memory. The arrows specify the flow of the operation. In the first row of the figure showing the CAM, a partial addition operation is performed on the first bits of $\mathrm{A}$ and $\mathrm{B}$ while $\mathrm{Cr}$ stores the carry bit. Therefore, the mask register is set as logic-1 for $\mathrm{Cr}$ and the first $\mathrm{A}$ and $\mathrm{B}$ columns. The second row of the figure similarly corresponds to the addition on the second bits. After each compare, the matched rows are tagged with logic-1, as indicated in its tag register. Then, the corresponding LUT entry (shown in the write column) is written only to the masked cells of the matched rows. For example, in the first table, "011" is searched for in $\mathrm{Cr}, \mathrm{B}$, and A columns, respectively. The firstrow matches by indicating a logic-1 in its tag register. As a result, logic- 0 is written for the $\mathrm{B}$ column, and logic- 1 is 


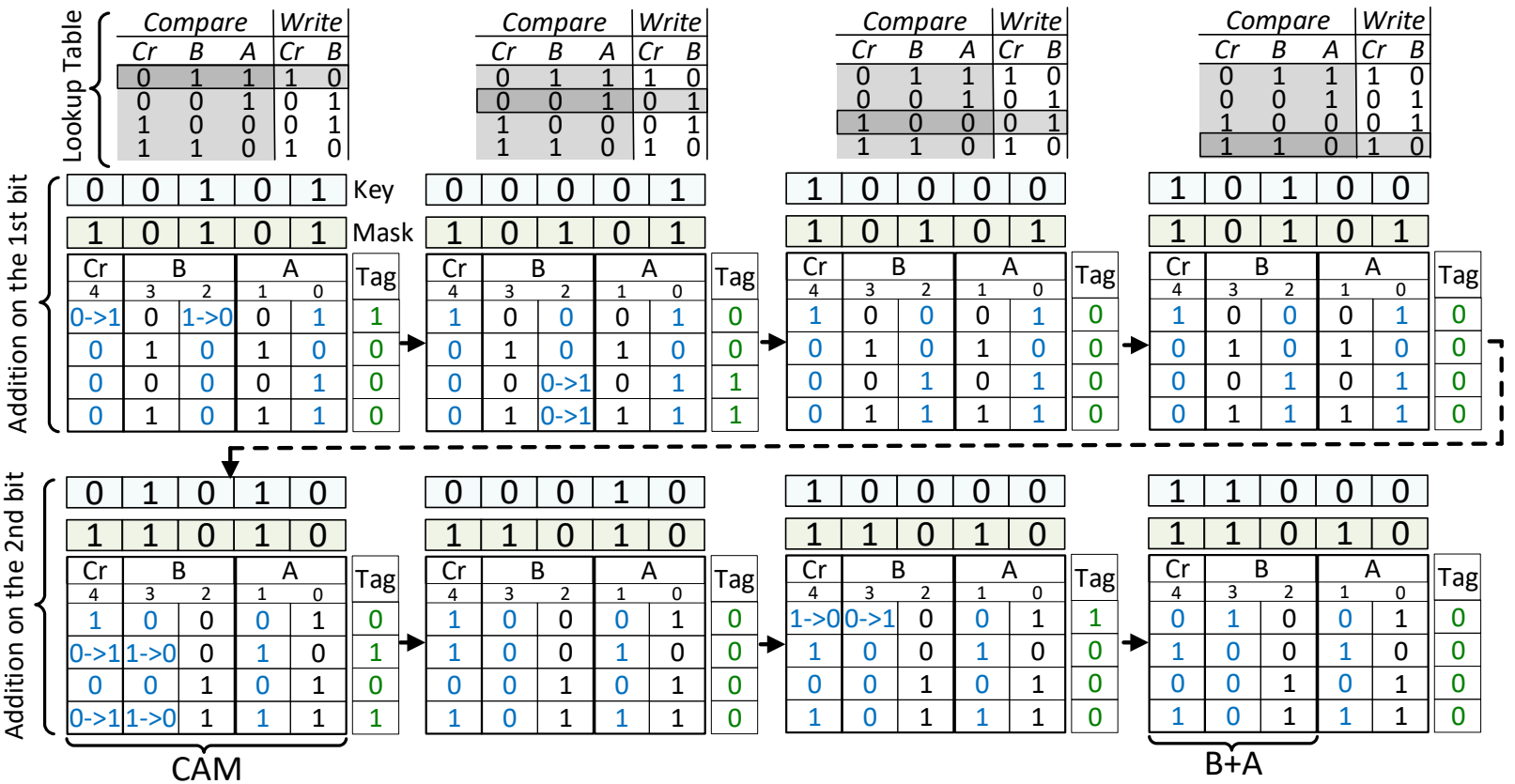

Fig. 2: An example vector addition operation $(B=B+A)$ in the $A P$ using associative computing principles

written to the Cr column. Normally, this operation represents a full adder for the combination of $0+1+1$, where the carry is logic-1, and the result is logic-0 (i.e., "10" as together). By applying all the LUT entries on each bit location, column-wise full addition is performed. The process is repeated for all the passes in the prescribed order shown in the figure. Finally, the value stored in $\mathrm{Cr}$ and $\mathrm{B}$ becomes $[2 ;-4 ; 1 ;-3]$, equals to $\mathrm{B}+\mathrm{A}$ (i.e., $[1+1 ;-2+-2 ; 0+1 ;-2+-1])$. Even though the figure shows an operation on a 4-element vector, this operation can be applied to hundreds of AP rows as concurrent. In general, adding two vectors that are m-bit wide takes $8 \mathrm{~m}$ cycles $(4 \mathrm{~m}$ compare and $4 \mathrm{~m}$-write), independently of the vector size as long as the vector fits into the memory. All other arithmetic and logical operations, such as subtraction, multiplication, etc., can also be performed in the memory as parallel by utilizing their corresponding LUTs [13], [14]. In short, these operations are performed by applying the corresponding operation's LUT on CAM consecutively. The controller part of an AP in Figure 1 is responsible for driving the CAM regarding the performed instruction. For vector operations on massive data, in-memory processing eliminates the memory access costs and provides a great performance advantage by its SIMD-like processing on each memory row.

In terms of performance, APs require a large amount of data to outperform traditional processors [14]. For this reason, their best usage is in applications that have an inherent SIMD (single-instruction multiple-data) computational pattern. Fast Fourier transform (FFT) [15], DNA sequence alignment [16], stencil [17], and matrix multiplication [18], [19] are some example applications that can benefit from AP. In an analogy, APs can be considered as a next step on the path of the CPU (central processing unit) to GPU (graphical processing unit) transformation. Compared with CPUs, GPUs have simpler processing cores; however, they are throughput optimized on the contrary to CPUs, which are latency optimized [20]. Figure 3 illustrates the architectural comparison of these three processors. Compared to GPUs, APs have much simpler cores (i.e., a single memory row), yielding a higher throughput since more cores can be placed in a chip. Additionally, its overall controller is smaller and simpler, which only includes the LUTs for the operations, and basic logic to apply these LUTs to the key and mask registers in order [13].

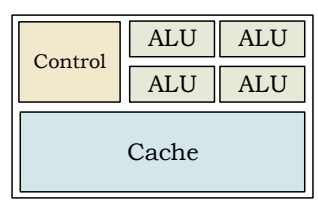

(a) CPU

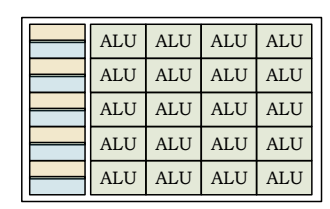

(b) GPU

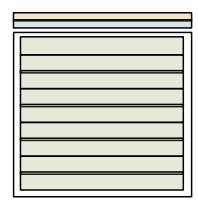

(c) $\mathrm{AP}$

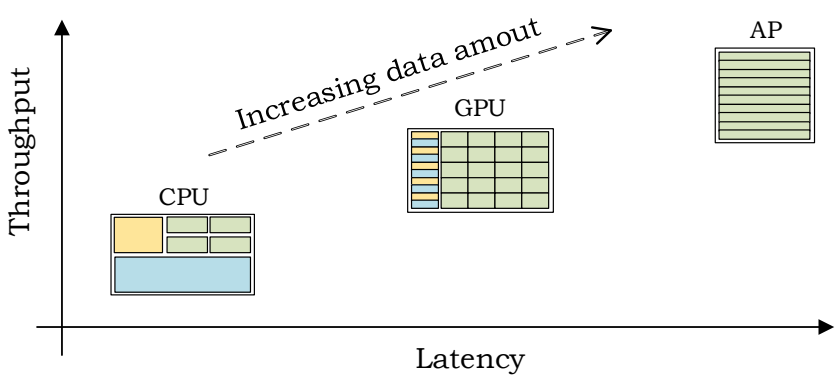

(d) Comparison

Fig. 3: Comparison of CPU, GPU, and AP

\section{B. Convolutional Neural Networks (CNN)}

Figure 4 shows the general architecture of a convolutional neural network. A typical CNN architecture consists of interleaved stages of convolution (conv) and activation layers followed by fully connected and classification layers. Normalization and pooling layers can also be used optionally. 


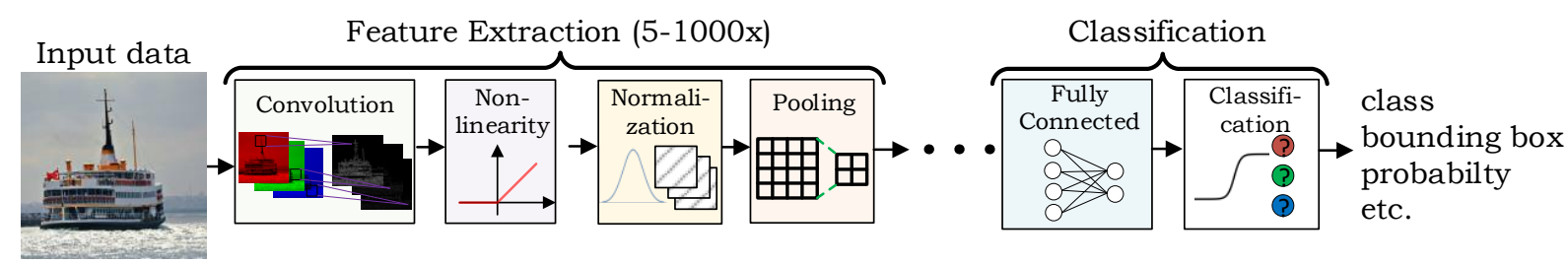

Fig. 4: Architecture of a convolutional neural network (CNN)

In the convolution, a 2D filter (kernel) is applied to the 2D data (i.e., activations) to extract the features. At each level, the convolution layer extracts higher-level features than the previous ones. These filters generally consist of hundreds of channels. After convolution, a nonlinear activation function is applied. The most widely used one is ReLU, which is the most computationally efficient [21]. The recent trend in $\mathrm{CNN}$ models is going deeper with more convolution layers, and the ratio between the number of convolution and fully connected layers gets larger. Convolution layers become the dominant part of overall performance and energy consumption. According to published statistics, $80 \%$ of the energy is spent on convolution layers versus fully-connected layers, and convolution operation in CNNs consumes more than $90 \%$ of the overall computation cycles [4], [22]. For CNNs, the bottleneck for processing is in the memory access since each multiply-accumulate (MAC) operation requires three memory reads and one memory write. Considering the fact that memory access consumes several orders of magnitude higher energy than computation [11], a memory-centric implementation of $\mathrm{CNN}$ architectures becomes vital.

Over the years, $\mathrm{CNN}$ architectures have become more and more complex. As a consequence, efficient hardware architectures are required to manage this complexity. In many applications, CNN inference should be carried near the sensors, which is referred to as edge computing [23], where low power operation is critical, especially in battery-operated scenarios. For critical approaches such as autonomous vehicle and drone navigation, local processing is essential since sending the data to the cloud is not feasible due to latency and possible reliability problems in the communication channels [24]. In the literature, there are many studies on CNN hardware architectures apart from the traditional CPU or GPU based solutions. All these architectures aim to accelerate convolution operations by optimizing memory usage and MAC operations. According to the literature survey study in [4], these architectures are classified into four groups as weight stationary, output stationary, no local reuse, and row stationary based on their data handling characteristics. The weight and output stationary architectures aim to optimize the energy consumption due to reading weights or partial sums of MAC operations, respectively [25]-[27]. On the other hand, row stationary architectures aim for more comprehensive optimization of weights, activations, and partial sums all together [28]. No local reuse (NLR) approaches aim to maximize global memory since it is more area efficient than local memories. In addition to these digital architectures, convolution operation can also be implemented by using SRAM or resistive memories (e.g., memristor, STT-RAM) to perform the MAC operation as analog [29], [30]. Even though analog computation is more energy-efficient, it is hard to program analog circuits, and they show lower accuracy due to electrical noise and inherent variability. Additionally, the fabrication process of emerging semiconductor devices is not mature yet. Therefore, most of the studies focus on small networks or only simulations [31][33].

This study proposes a traditional CMOS-based hardware architecture for the convolution accelerator to put effort into solving the aforementioned problems of convolution processors. The contribution of the study to the research community can be summarized as follows:

- For the first time, a novel AP-based in-memory convolution accelerator (IMCA) is proposed, which can achieve high energy efficiency and optimal computational density by bringing the computation inside the storage.

- Unlike the other accelerators, which generally take the absolute value sparsity into account, the IMCA is capable of regarding bit-level sparsity, which facilitates the finegrain optimization for performance and power.

- The cost of some AP operations such as multiplication, absolute value is reduced by proposing more efficient alternatives. The new operations both consume less area, power as well as show better performance.

- It is shown that the proposed architecture supports approximate computing inherently and dynamically without any extra cost, which shows the superiority over other CNN architectures.

\section{ACCELERATOR ARCHITECTURE}

The proposed accelerator (IMCA) is based on associative in-memory processing. The overall architecture consists of multiply-accumulate units aligned in a pipelined way. Furthermore, the architecture includes some additional novel mechanisms such as constant multiplication, bit-level sparsity, and dynamic approximate computing introduced in this study. The following subsections detail the overall architecture.

\section{A. In-memory Convolution}

Convolution operation on the IMCA is performed through associative processing principles. The architecture uses fixedpoint arithmetic. Figure 5 shows the overall pipelined IMCA architecture where the activations are stored on the CAM rows, and the kernel weight for the corresponding MAC operation is stored in a register inside the controller. Each stage of the IMCA performs a MAC operation on these activations with its corresponding filter weight. In a $k \times k$ filter, there are $k^{2}$ filter weights. Since each stage of the IMCA performs a MAC 


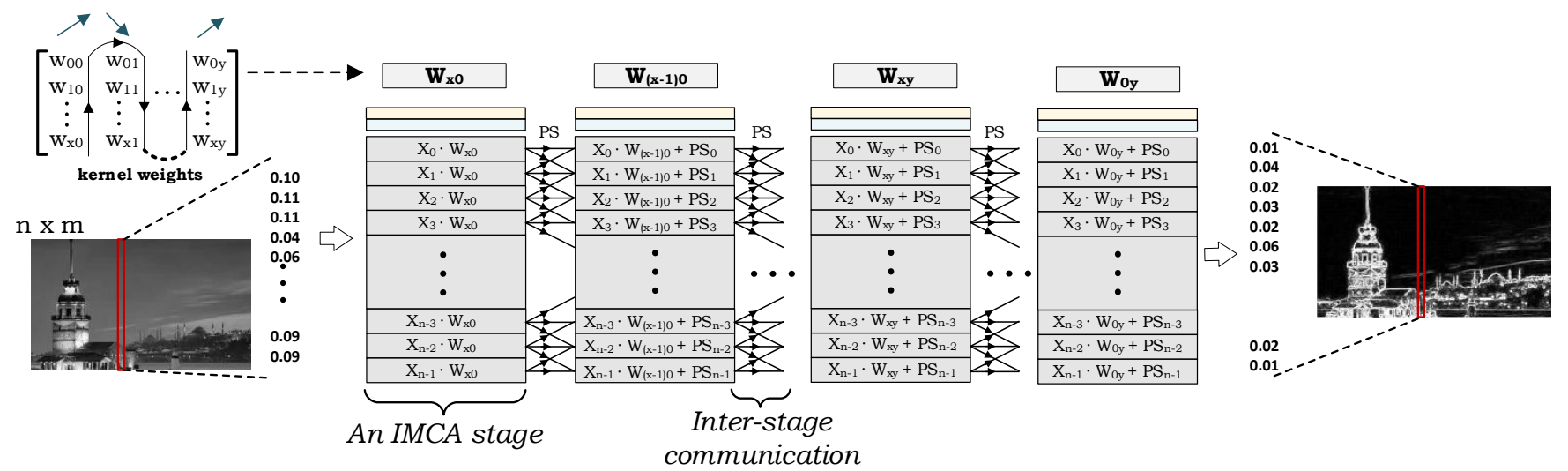

Fig. 5: Architecture of in-memory convolution accelerator (IMCA)

operation on a kernel weight, the total number of the required stages equals to $\mathrm{k}^{2}$. Additionally, an extra stage can be added for buffering the computed activations if the number of stages is not enough for the filter weights (i.e., filter size $>\mathrm{k}^{2}$ ). While first $\mathrm{k}^{2}$ stages are responsible for MAC operation for each filter weight, the optional last stage stores the output values either to be read by an outer processor or for partial sums with the results of other activations. The figure also shows the placement of kernel weights through the pipeline stages. The placement starts from the bottom of the first column

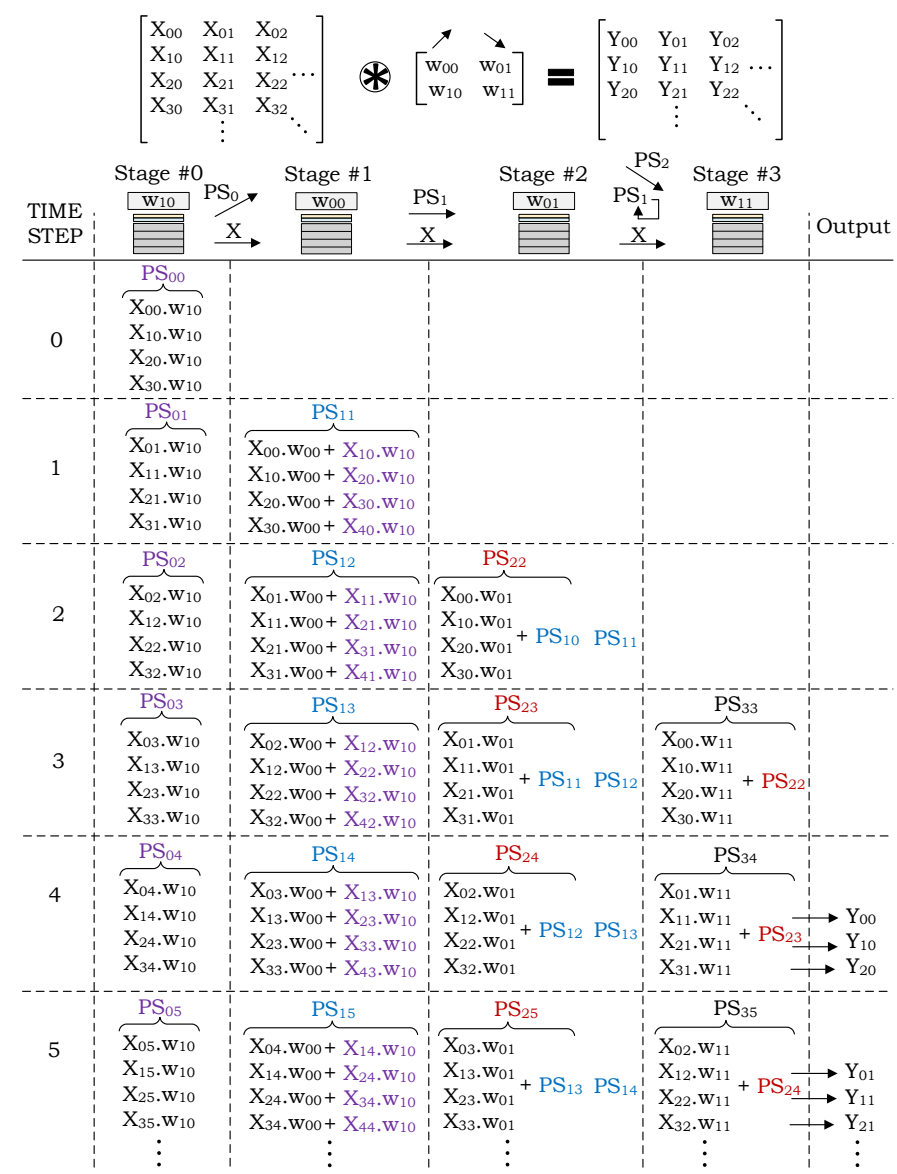

Fig. 6: Convolution through the IMCA stages and follows an s-shape pattern through the last column. This placement is required to optimize the data flow between the CAMs. This placement is also effective on the communication pattern between the stages (i.e., forward, up, or down). Each column of the filter has alternating up and down communication patterns, and only the first element of the column has the forward pattern for the partial sums (PS). The weights are loaded only once at the beginning of the convolution operation on $2 \mathrm{D}$ input activations. While input activation is fed to the IMCA column by column, the same filter weights are reused until the next convolution operation with a different filter. The first stage gets the input data from the outside processor or another data source (e.g., sensor) as sequential. During this stage, the IMCA is initialized with a column of the 2D input data (e.g., an image column). This sequential initialization operation is done by using a shift register, which activates a row of the CAM at a time, in order. Whenever a row is activated (i.e., write enable signal is asserted), the incoming data is written. This methodology eliminates the need for areainefficient address decoder and encoder circuits. In order to obtain an area-efficient implementation, the tag registers in traditional AP architecture are modified to function as a shift register as well. Figure 1 shows this detail also where each tag register is connected to the next one to form a chain. The outer processor sends the data as synchronized with the tag register so that the tag register shifts by one bit at each clock cycle whenever new data comes. In the first stage, there is no accumulate operation, only multiplication. Starting from the second stage, both multiplication and accumulation (MAC) operations are carried on the data coming from the previous stage. Even though the initialization of the IMCA is performed row-wise, the communication between the stages is carried out column-wise. After performing a MAC operation in an IMCA stage, the partial summation results and input column are sent to the next stage. An interconnection switch handles communication between the IMCA stages. A general-purpose interconnection switch between two CAM stages [34] requires $\mathrm{n}^{2}$ configuration cells and supports $\mathrm{n}$ ! different communication patterns. This constitutes an interconnection switch which is more complex than the CAM itself, where CAM size is $\mathrm{n} \times \mathrm{m}$ and $m$ (i.e., number of columns) is generally much smaller than $n$ (i.e., number of rows). Additionally, the required 
control unit to manage such a huge number of combinations becomes very complex and intractable. On the other hand, the proposed IMCA processor requires only three different communication patterns, which are directly forward, one row up, and one row below due to the optimized placement of the kernel weights as illustrated in Figure 6. The three-state buffers handle this communication, as shown in the detailed circuit diagram in Figure 13a. This scheme facilitates the usage of limited reconfigurable communication interconnect between the stages that provides enough flexibility for convolution operation and low implementation cost.

$$
\mathrm{PS}_{\mathrm{st}}=\left[\begin{array}{c}
\mathrm{X}_{0(\mathrm{t}-\mathrm{s})} \\
\mathrm{X}_{1(\mathrm{t}-\mathrm{s})} \\
\vdots \\
\mathrm{X}_{\mathrm{n}(\mathrm{t}-\mathrm{s})}
\end{array}\right] \cdot \mathrm{w}_{\mathrm{S}}+\mathrm{PS}_{(\mathrm{s}-1) \mathrm{x}} \quad \text {,where } \mathrm{x}=\left\{\begin{array}{l}
\mathrm{t}-2, \text { if } \mathrm{s} \% \mathrm{k}==0 \\
\mathrm{t}-1 \text {, otherwise }
\end{array}\right.
$$

Figure 6 explains convolution operation on the IMCA explicitly. In the figure, the filter has a kernel size of two for simplicity. The figure follows a convention where $P S_{s t}$ shows the partial summation result of MAC operation on stage $s$ at time step $t$. The computed partial sum in a stage is forwarded through the last stage to obtain the resulted activation at the last stage. The figure shows the partial sums generated in a stage at a specific time in the same color. For example, $P S_{22}$ is generated in stage \#2 at the time step of 2. This partial sum moves to the next stage at time step 3 to generate the partial sum in the following stage (i.e., $P S_{33}$ ). $X$ represents the column vector of input activations and always moves forward between the stages. Equation 1 shows the performed computation for the partial sum in an IMCA stage, where a MAC operation is performed with the same filter weight of the corresponding stage (i.e., $w_{s}$ ). In all the stages except the ones corresponding to the first column weights of a kernel matrix, the accumulate operation is performed between the product and partial sums from the previous stage and time step. For the first column weights of a kernel matrix (e.g., the third stage), the accumulate operation is performed with the partial sums coming from the previous stage and two previous time steps. The figure also shows data movements between the stages. For example, while the result of stage $\# 0\left(\mathrm{PS}_{0 \mathrm{t}}\right)$ shifts one row up, $\mathrm{X}$ moves forward between stage \#0 and stage \#1. X (i.e., an image column) always moves forward through all pipeline stages. Equation 2 formalizes the communication pattern for partial sums between the stages.

$$
\mathrm{PS}_{\mathrm{st}} \leftarrow\left\{\begin{array}{c}
\mathrm{PS}_{\mathrm{st}} \quad \text { if }(\mathrm{s}+1) \% \mathrm{k}==0 \\
{\left[\begin{array}{c}
\mathrm{ps}_{1} \\
\mathrm{ps}_{2} \\
\vdots \\
\mathrm{ps}_{(\mathrm{n}-1)} \\
---
\end{array}\right]_{\mathrm{st}} \text { else if } \mathrm{shift}==} \\
{\left[\begin{array}{c}
--- \\
\mathrm{ps}_{0} \\
\mathrm{ps}_{1} \\
\vdots \\
\mathrm{ps}_{(\mathrm{n}-1)}
\end{array}\right]_{\text {st }} \text { else if } \text { shift }==\searrow}
\end{array}\right.
$$

The latency of the processor is $k^{2}$ steps, where a step consists of a MAC operation and data movement to/from

that stage. After the latency, the system outputs a computed convolution results across a column at each time step.

\section{B. CAM Optimization}

The IMCA relies on in-memory computing based on a CAM. The operands on which the in-memory operations are performed are stored in SRAM-based CAM cells. The overall memory size must be enough to store all the operands and perform the necessary operations. On the other hand, one of the biggest problems of volatile memory technologies such as SRAM is the static power consumption where the transistors in the cells leak the current while keeping the data. The leakage power constitutes a big portion of the overall energy consumption of an SRAM memory [35], [36]. The total amount of leakage is directly proportional to the CAM size. Therefore, the proposed IMCA architecture should perform convolution operation by using a minimum number of CAM columns within the shortest time to reduce the overall leakage. For this reason, an effort has been made to optimize some operations by both decreasing their area usage and latency. The constant multiplication operation introduced in the next subsection (Section III-C) positively improves the leakage energy by decreasing the latency of multiplication operation. Furthermore, it decreases the CAM size since there is no need to store the kernel weights inside the CAM. For further reduction both in the static power consumption as well as the area, some operations can be performed as in-place instead of traditional out-of-place execution [14], [37]. In in-place operations, the results of the operations are overwritten to one of the operands (e.g., $\mathrm{B}=\mathrm{B}+\mathrm{A}$ ). Out-of-place operations require additional columns to store the result (e.g., $\mathrm{R}=\mathrm{B}+\mathrm{A}$ ). As seen in Figure 10, the addition operation is already performed as in-place. A possible instruction to be performed as inplace is absolute value operation, which is needed for signedmultiplication. The operation is used two times to get the absolute value of input activation and convert the multiplication result to its original sign. Instead of using traditional absolute value operation where the result is written to another place, the in-place absolute value operation is proposed by utilizing the hardware optimization in [38] where the result is written in the same place as the operand. Table I shows these two LUTs for absolute value operations performed as bit-wise. As shown in Table Ia, traditional LUT for absolute value operation $(\mathrm{R}=|\mathrm{A}|)$ requires a special ordering as indicated in the comment column. The table simply copies $A$ to $R$ if it is positive $(S=0)$. Otherwise, it performs 2's complement on $\mathrm{A}$ for its absolute value. In the table, $\mathrm{F}$ stands for the flag bit, which becomes logic-1 whenever a logic-1 is seen in A's corresponding column. When it becomes logic-1, the LUT

TABLE I: LUTs for absolute value operations in APs

(a) AbsoluteValue

\begin{tabular}{ccc|ccl}
\hline$F$ & $S$ & $A$ & $F$ & $R$ & Comment \\
\hline 0 & 0 & 1 & 0 & 1 & 1stPass \\
0 & 1 & 1 & 1 & 1 & 4 thPass \\
1 & 1 & 0 & 1 & 1 & 2ndPass \\
1 & 1 & 1 & 1 & 0 & 3rdPass \\
\hline
\end{tabular}

(b) AbsoluteValueIP

\begin{tabular}{ccc|cc}
\hline$F$ & $S$ & $A$ & $F$ & $A$ \\
\hline 0 & 1 & 1 & 1 & 1 \\
1 & 1 & 0 & 1 & 1 \\
1 & 1 & 1 & 1 & 0 \\
\hline
\end{tabular}




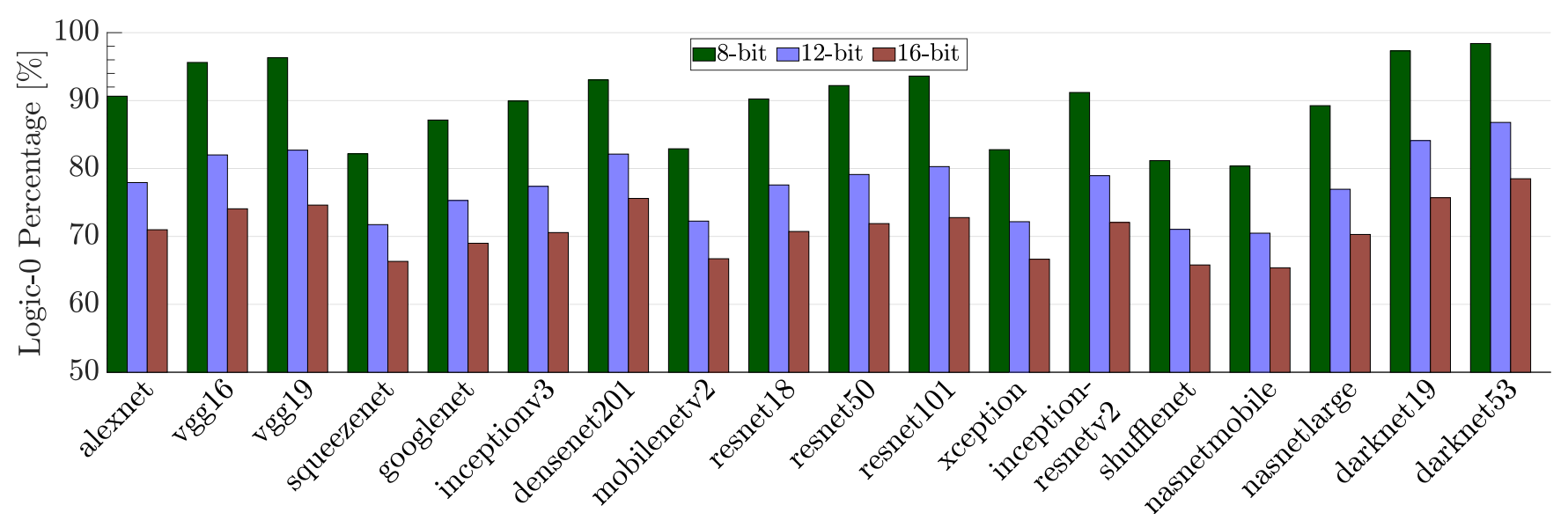

Fig. 7: Percentage of logic-0 in unsigned binary representation of the filter weights for 18 pre-trained CNNs (i.e., 1-k/m where $\mathrm{m}$ is the total bitwidth and $\mathrm{k}$ is the number of logic-1s)

sets the $\mathrm{R}$ as the reverse of $\mathrm{A}$ for the remaining bits. The approach in [38] provides a mechanism which excludes the matched rows in the next compare cycle within a LUT pass, therefore releases the requirement for LUT ordering as well as it avoids the miswrites during a LUT pass. Table II shows the comparison of the proposed operation with the previous version. According to the table, the proposed new operation (AbsoluteValueIP) provides an average of $48 \%$ improvement in the area. Furthermore, it increases the performance of the operation by an average of $36.5 \%$.

TABLE II: Comparison of the proposed absolute value operation with the traditional one

\begin{tabular}{|r|c|c|c|}
\cline { 2 - 4 } \multicolumn{1}{c|}{} & AbsoluteValue [14] & AbsoluteValueIP & $\begin{array}{c}\text { Improvement \% } \\
\text { (16-bit) }\end{array}$ \\
\hline Area Requirement & $2 \mathrm{~m}+2$ & $\mathrm{~m}+1$ & $48 \%$ \\
\hline \# of compare cycles/bit & 4 & 3 & $40 \%$ \\
\hline \# of write cycles/bit & 4 & 3 & $33 \%$ \\
\hline
\end{tabular}

\section{Constant Multiplication and Bit-level Sparsity}

In APs, the theoretical complexity of multiplication operation is $\mathcal{O}\left(\mathrm{m}^{2}\right)$, where $\mathrm{m}$ is bitwidth of the multiplier, and multiplicand [34]. This is because a partial addition operation is performed for every bit of the multiplier operand [13]. Basically, this operation adds the partial result to multiplicand if the corresponding multiplier bit is logic-1. On the other hand, if the bit is logic-0, it simply skips all the write cycles since there will be no match at the end of a compare operation. This operation is crucial for many applications such as FFT, Fast Walsh-Hadamard transform, etc., when the multiplier is variable through the CAM rows [13], [15]. On the other hand, in CNNs, the same weights are applied to a frame during a convolution operation by performing constant multiplication. Convolution operations on the IMCA require vectorial multiplication where data through the CAM rows are multiplied with the same constant (i.e., the kernel weight) as shown in Figure 6. If a binary factor $\left(2^{\mathrm{n}}\right)$ exists for the inputs or weights, the shifting operation can be applied to find the exact value. This operation takes a single cycle since only fixed-point representation is changed [14].
Figure 7 shows the logic- 0 percentage of the all convolution weights of 18 different pre-trained CNN models where the bitwidth representing the weights varies between 8-16 bits. The figure shows the statistics of around 390 million unsigned weights used by the popular CNNs, where the average $71.0 \%$ of the binary representation of the weights consists of logic-0s in their 16-bit representations, resulting in a sparse multiplication operation. In an 8-bit representation, which is more commonly used by the inference engines, this ratio goes up to $89.7 \%$. These findings create a perfect opportunity for the associative in-memory convolution engine where the engine simply skips logic-0 bits of the multiplicand (i.e., weight) during the costly multiplication operation. Regarding this need, the architecture of the APs is modified to support faster and more energy-efficient in-memory multiplication operation.

During the convolution operation, the controller unit checks the bit of the constant (i.e., kernel weight) and performs the partial addition operation if it is logic-1. The approach requires an additional comparator inside the controller, which has a negligible cost compared to the overall architecture. The approach resembles loop unrolling in traditional compiler designs [39]. In this way, a considerable area and static power savings become possible since there is no need to store the same kernel weights across the rows in the CAM. On the other hand, traditional $\mathrm{CNN}$ architectures require to multicast the same kernel weight to multiple processing elements. Figure $8 \mathrm{~b}$ shows an example case for bit-level sparsity during the traditional multiplication operation, where the activation $x$ is multiplied by the weight $w$. The weight includes two logic-0s

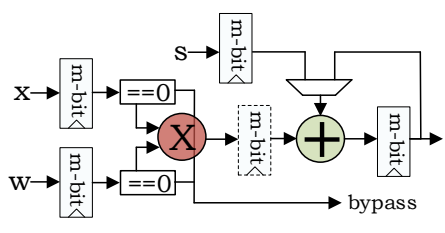

(a) Traditional MAC operation

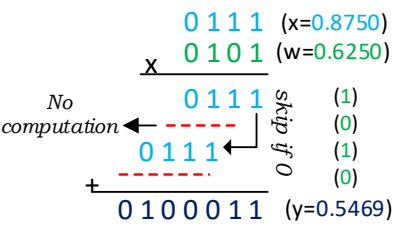

(b) Bit-level sparsity
Fig. 8: Traditional MAC operation and bit-level sparsity in the IMCA 
in its representation (2nd and 4th bit); therefore, whenever the $w$ 's corresponding bit is logic- 0 , the partial addition operation is skipped for the sake of both performance and energy. Since multiplication is a quadratic operation in the AP, the complexity of multiplication decreases to $\mathcal{O}(k \cdot m)$ where $\mathrm{k}$ is the number of logic-1s in the weight and $\mathrm{m}$ is the bitwidth of the feature and weight. If the binary digit distribution is accepted as uniform $(2 \mathrm{k}=\mathrm{m})$, this modification provides a $2 x$ speedup. Further to that, Figure 7 also implicitly exhibits speedup and energy saving since they are directly proportional to the logic-0 percentage (i.e., 1-k/m). As presented in the figure, the ratio of $\mathrm{m}-\mathrm{k}$ (i.e., logic-0 percentage) over $\mathrm{m}$ changes between $65.3 \%$ and $98.4 \%$ with an average of $78.8 \%$. This corresponds to an energy-saving proportional to this rate due to the faster processing. Figure 9 shows the required number of cycles (compare and write) to perform the multiplication operation between the input activation and the kernel weights ranging from 8-bit to 16-bit. The figure shows the results of traditional multiplication on the AP and the proposed constant multiplication on different pre-trained CNN weights. The average speedup that the proposed multiplication for IMCA achieves varies between $3.5 \mathrm{x}$ and $15.5 \mathrm{x}$. On the other hand, a traditional CMOS-based multiplier (Figure 8a) used in other convolution engines cannot get benefit from this feature and functions the same for all weights whether it is all logic zeros except the LSB (i.e., 0.00000001) or all logic1s (i.e., 0.11111111). However, some architectures include the energy reduction technique to bypass the multiplication operation if the weight or activation is 0 (i.e., all bits are 0) [28], [40].

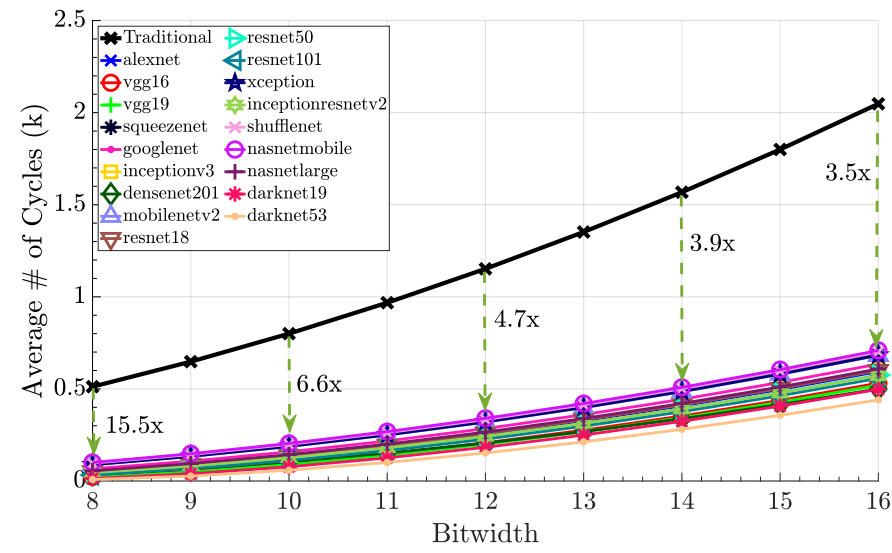

Fig. 9: Number of cycles required for the multiplication

Figure 10 shows MAC operation on the CAM rows of IMCA step by step in a total of five steps when the proposed CAM optimizations and constant multiplication are employed. The figure details the operations performed in a single stage of Figure 6. Through the CAM rows, all MAC operations are performed simultaneously, resulting in a run-time that depends on the bitwidth rather than the length of the processed vector as long as it fits into the memory. In the figure, $x_{i, j}$ and $s_{i, j}$ represent the input activation and the result of MAC operation in row $j$ of $i^{t h}$ stage. The $s b$ indicates the sign bit of the multiplication result. Since multiplication operation is performed on unsigned numbers, firstly, the absolute value operation (AbsIP) is performed on input activation. Constant multiplication operation (MultConst) is performed with the same filter weight $\left(w_{i}\right)$. After the multiplication operation, another absolute value operation is carried over to obtain the signed result. Lastly, in-place addition operation (AddIP) is performed to obtain the final result $s_{i, j}$.

\section{Dynamic Approximate Computing}

Training of DNNs requires computation at high precision since gradient operations must be performed precisely to optimize the overall architecture weights. For this reason, the training phase is generally performed at high precision by representing numbers in floating-point in GPUs [41], even though there are some studies performing training with little to no loss in accuracy at 16-bit fixed-point [42]. On the contrary, the inference step of DNNs does not require such amount of accuracy, so can be performed by fixed-point numbers with fewer bits ranging from 8-16 bit [43], and even in binary [44]. The study in [24] shows that the number of integer bits required for MAC operations during $\mathrm{CNN}$ is 8 bits for $99.12 \%$ weights and 16 bits for $0.01 \%$. On the other hand, some architectures may require better precision, especially if it is mission-critic (e.g., healthcare). Therefore, the recent GPU architectures provide reconfigurable precision for training and inference and support mixed-precision computing [45], [46].

In the IMCA, operations are performed bit-wise, starting from the least significant bit (LSB) towards the most significant bit (MSB). The locations of the LSBs represents the accuracy (bitwidth) of the numbers, and it can be inherently changed by modifying the instruction on the controller. While operations are performed over 16-bit, at any time, the precision can be decreased to 8-bit and later increased to 12-bit. Furthermore, each stage in the IMCA can perform operations on different bitwidth. In this way, while low-level features are obtained in high precision, higher-level features can be obtained in a lower precision as a preferred methodology in CNNs. The architecture naturally provides a mechanism of dynamic approximate computing, which is very useful for DNN architectures. Figure 11 shows results for approximate computing in the IMCA. The figure presents the GOPS and accuracy results when the bitwidth of the IMCA is changed for MAC or only unsigned multiplication operations. For accuracy results, the reference is 16-bit computation. For the MAC operation, the system changes the output precision to a specified bitwidth. For example, two 8-bit numbers are multiplied, and a

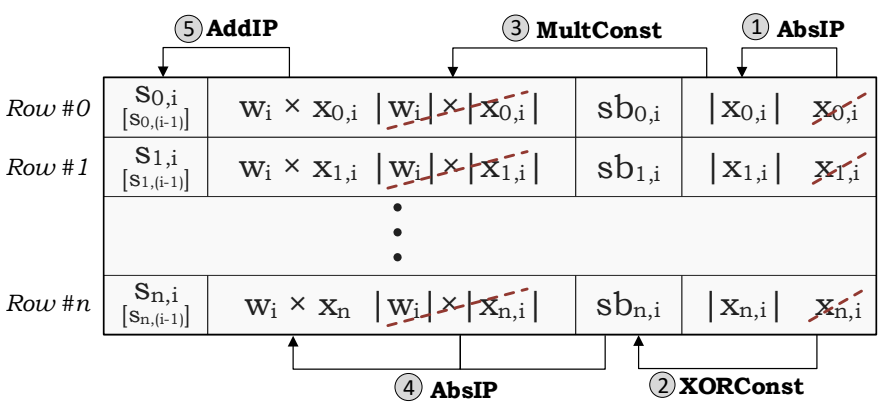

Fig. 10: MAC operation on the $\mathrm{i}^{\text {th }}$ stage of the IMCA 
16-bit number is obtained. Then, this number is trimmed back to 8-bit during accumulation. On the other hand, the results belong to the multiplication corresponds to the case where the output is always 16-bit. However, the input numbers are trimmed to the specified bitwidth only during multiplication. For example, two 12-bit numbers are multiplied, and a 24bit number is obtained. This number is trimmed back to 16-bit, and accumulation operation is performed over 16-bit. According to the figure, performing approximate computing only during multiplication operation results in an advantage in GOPS with better accuracy compared to overall computing (MAC). Moreover, this method keeps the output at full precision. For example, at the full 16-bit precision output with 99.6\% accuracy, 2.70x GOPS improvement can be obtained when the multiplication operation is performed over 8-bit.

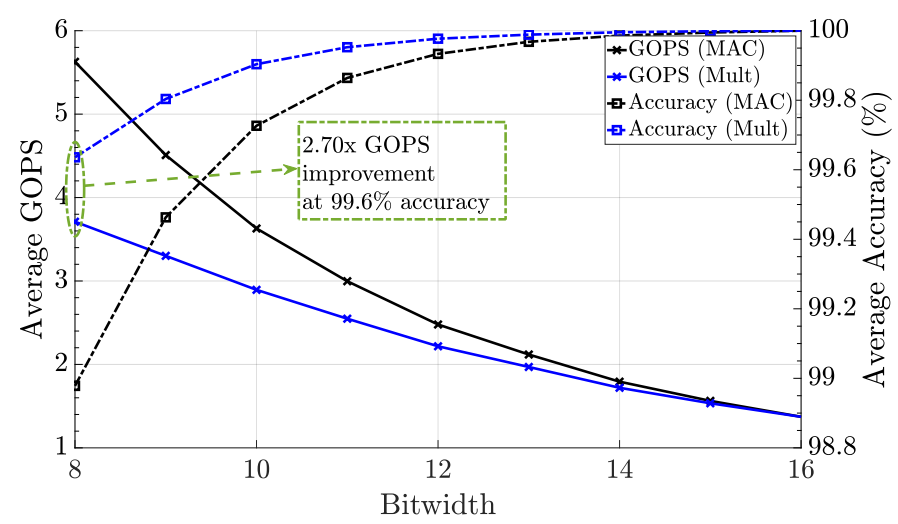

Fig. 11: Average GOPS and accuracy metrics of multiplyaccumulate (MAC) and multiply operations in a single IMCA stage (256-row) with respect to variable bitwidth

\section{E. System-level Architecture}

Even though the most costly operation in CNNs is convolution, CNNs also include more various units like activation, pooling, fully connected layers, etc. Thus, IMCA can be used as an accelerator more usefully whenever an efficient/parallel convolution operation is required during the overall flow of a $\mathrm{CNN}$ benchmark. Figure 12 shows an illustrative processing system architecture where the IMCA is directly coupled to processor and memory to be used as an accelerator near a processor. In this way, whenever the CPU encounters a convolution operation (e.g., nn.Conv2D() in PyTorch), it can offload this operation to the IMCA. In the figure, the top diagram shows the $2 \mathrm{D}$ convolution operation between the $m, c$-channel filters (W), and c-channel input activation (I). During the convolution, each filter is convolved through the input feature's channels and generates a single 2D output activation. Each $m$ filter generates a separate output activation with a total of $m$ output activations. At the beginning of the operation, input activations of the convolution to be filtered can be copied to the AP from the main memory or CPU through the dedicated buses. The CPU can initiate the operations on the IMCA. The accelerator itself can consist of multiple IMCA units, which are organized in a two-dimensional space. The figure shows the overall architecture, which consists of $c$ tiles, and each tile has $m$ IMCA units. The data (activation, filter

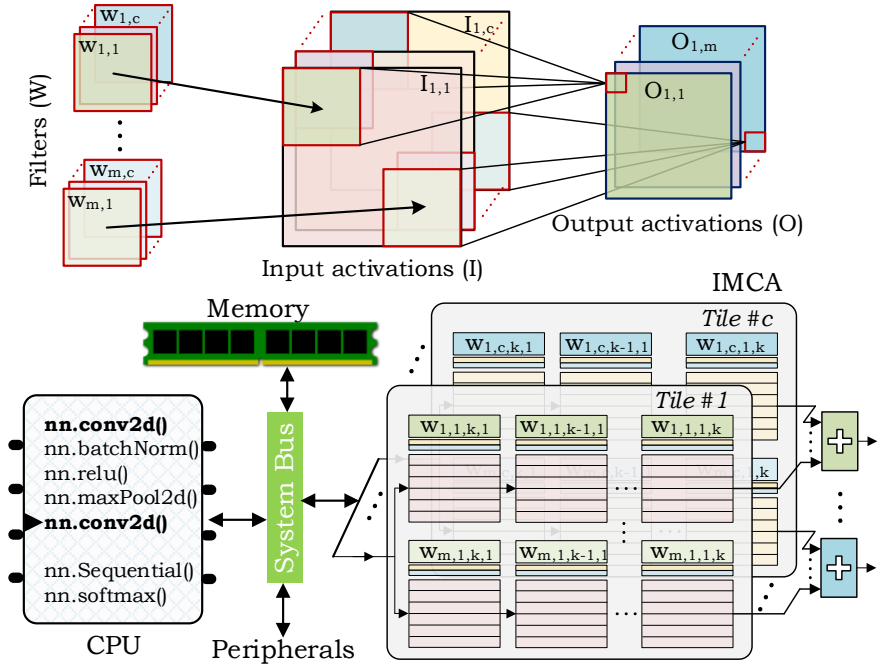

Fig. 12: Convolution operation on the IMCA and overall system architecture where the corresponding data for the convolution operation (filters and activations) shown in the same color

weights, and output) placement are shown in the same color as in the convolution diagram at the top for better understanding. In a tile, the same data are transferred to all IMCA units. However, a different filter is applied to each input activation through the pipelines (i.e., IMCA unit). In this way, input activation reuse is achieved. The total number of tiles depends on the number of channels. At the end of the convolution, the corresponding output activations across the tiles are summed to compute the final activation. The most important benefit of IMCA here is that since all the activations and weights are stored inside the processor, no memory access is required outside compared to the traditional architectures. In this way, the reuse of input activations, weights, and output activations is achieved. In the end, the results can be copied back to the memory or directly used by the processor. Even though the figure shows the perfect placement of a convolution operation on the IMCA where the number of channels and activations is the same as the processor dimensions, the perfect placement might generally not be possible since the sizes change through the CNN layers. In this case, a time-division multiplexing approach can be used to perform the convolutions in multiple runs. For instance, the maximum filter size depends on the number of MAC stages in an IMCA unit. Supposing the filter size is larger than the number of IMCA stages, in that case, an additional stage can be used for buffering, and the filter weights are replaced in the controller to perform the computations with the remaining filter weights.

IMCA can also be employed by tightly coupling it with the CPU in the same chip as in [47], or replacing the last level cache of a CPU by IMCA [34]. It can also be combined in the same chip with a memory to facilitate faster communication to eliminate the DRAM bottleneck. Furthermore, the proposed accelerator can be used as a subcomponent in the ASIC design of AI chips. Since the main target of this study is introducing a convolution accelerator on its own, the system- 
level architecture solutions are postponed to further studies for the sake of content constraints.

\section{EVALUATION}

For the evaluation of the IMCA, an in-house simulation framework is developed, which consists of both system-level and circuit-level simulators. The system-level simulator takes the input (2D image, kernel weights, etc.) together with the architecture features (bitwidth, approximate computing parameters, number of layers, etc.) and drives the SPICEbased circuit simulator iteratively as cycle accurate to obtain the accurate results. For the transistor models, 65-nm TSMC transistor models at TT (typical-typical) process corner are referenced. A low-power, sub-ns sense amplifier design in [48] is employed in the matching circuit of CAM. For energy reduction, the proposed techniques in [38] are exploited. The controller is implemented by Verilog. The CAM architecture is designed and verified on Synopsys Custom Compiler. CAM architecture is specifically optimized for associative computing,

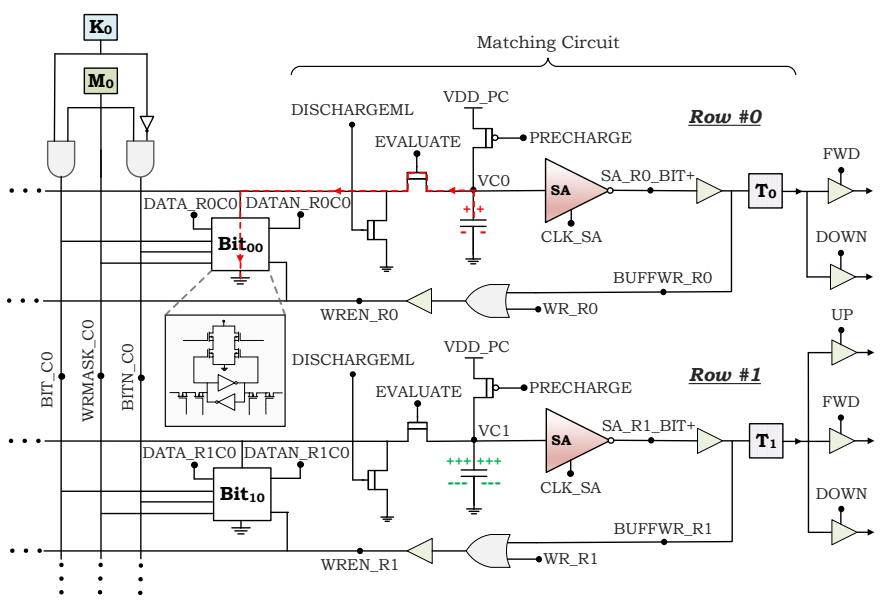

(a) Circuit block diagram
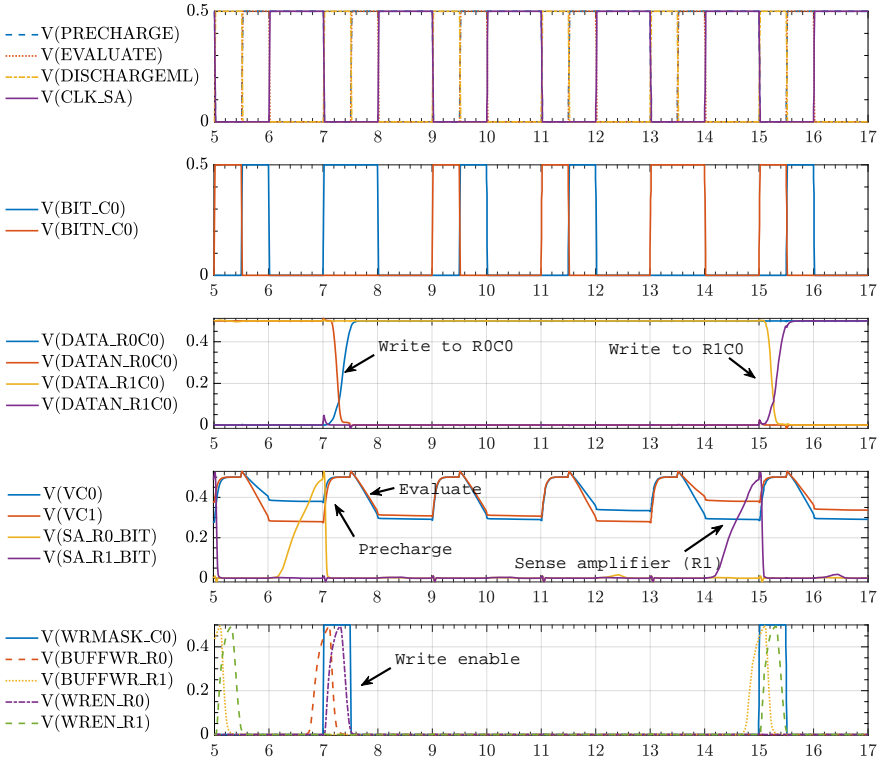

(b) Simulation waveforms

Fig. 13: Circuit block diagram and simulation waveforms of the AP circuit where a compare operation is done at most four columns. For this reason, the general-purpose memory models like CACTI [49] are not used. Figure 13a shows the circuit block diagram of the AP architecture taken as a base in the evaluations. Even though the implemented circuit is much more complex (e.g., there are buffer trees to drive the signals, the sense amplifier also has BIT- output, etc.), the figure shows the essential circuit diagram in the abstract. The parasitic line resistances and capacitances are also included in the circuit description to obtain more realistic results. Figure $13 \mathrm{~b}$ shows the simulation waveforms obtained from Synopsys Custom WaveView after the simulation. Overall system operates at 1 $\mathrm{GHz}$ clock frequency, where the compare operation takes $2 \mathrm{~ns}$ ( $1 \mathrm{~ns}$ for compare and $1 \mathrm{~ns}$ for sense amplifier). The waveform shows the six compare operations done consecutively between the $5 \mathrm{~ns}$ and $17 \mathrm{~ns}$ during a MAC operation. The waveforms in the figure correspond to the labeled locations in Figure 13a. In the first compare cycle (precharge \& evaluate), there is a match in row\#0, so the corresponding sense amplifier $\left(S A_{-} R 0_{-} B I T\right)$ results in logic-1. The write operation can be done during the precharge to hide the write time cost. Therefore in the following precharge cycle, the data of the cell $R 0 C 0$ is changed as logic-1. The same operation is done for $R 1 C 0$ also as a result of the compare operation between $13 \mathrm{~ns}-15 \mathrm{~ns}$ where the sense amplifier at the second row ( $\left.S A_{-} R 1_{-} B I T\right)$ outputs logic-1. Since the computation is performed directly inside the memory rows, unlike the traditional logic, a low-power operation is aimed at setting the voltage to $0.5 \mathrm{~V}$ without violating the functional integrity of $\mathrm{AP}$ operations. On the other hand, a low-voltage decreases the read speed of SRAM cells considerably. At this voltage, an SRAM can operate up to $150 \mathrm{Mhz}$ frequency [50]. Since convolution operation through the AP units does not require SRAM read operation except in the last stage to get the convolution result back, this low voltage does not constitute an impediment for the IMCA. On the other hand, an attainable frequency of 150 $\mathrm{MHz}$ is enough to read all the 256-row convolution results back in a pipelined system since an IMCA layer requires an average of 1184 compare cycles where each cycle takes 2 ns. Alternatively, the first and last stage CAMs can be driven at a higher voltage for a faster write/read operation if the number of required rows is higher. The results presented here reflect the overall performance of convolution, which is the most costly operation for AI accelerators. The results also include the data initialization cost to the accelerator. On the other hand, the cost of DRAM is not taken into account since it is an out of chip component and not considered in the other studies as well [51]. The study aims to present an accelerator architecture focusing on the convolution operation. Since the study proposes the convolution architecture, the system-wide evaluations are postponed for the future studies, rather presented the performance metric for single convolution operation on 2D data. The proposed accelerator can be used as a sub-component in any general processor architecture by combining with other units such as pooling, nonlinear activation, and normalization as indicated in Section III-E.

For the architecture configuration, the CAM size is set enough to perform convolutions on a maximum of 16-bit 


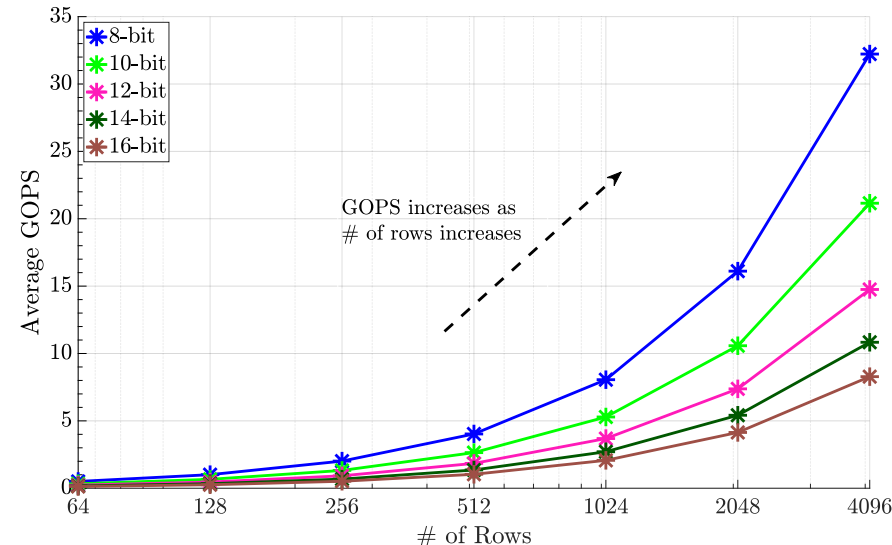

Fig. 14: Average GOPS of a single IMCA stage with respect to variable number of rows (size of the image) and bitwidth

features and filters. On the other hand, the architecture supports computation between 1-16 bits variable precision since it supports dynamic approximate computing. If the convolution is done on $\mathrm{m}$-bit numbers, the number of required columns is $4 \mathrm{~m}$; therefore, it is set as 64 . The number of rows is selected as 256 to perform the convolutions on the biggest feature sizes [52]. Therefore, each CAM has a size of $64 \times 256$ bits. The survey in [4] shows that the most modern CNN applications require a filter size of at most seven. Moreover, the trend tends towards decreasing the filter size by decomposition for computational efficiency and increasing the number of layers to extract better features [4]. Therefore, for the results provided, the filter size is fixed to $7 \times 7$, which requires 49 MAC stages for the proposed in-memory implementation. On the other hand, the effective throughput does not depend on the number of data rows as well as on the number of MAC stages. In other words, the other filter sizes also give almost identical results except for the area and the total energy consumption. In the results, each MAC is assumed as a combination of two operations (multiplication and addition).
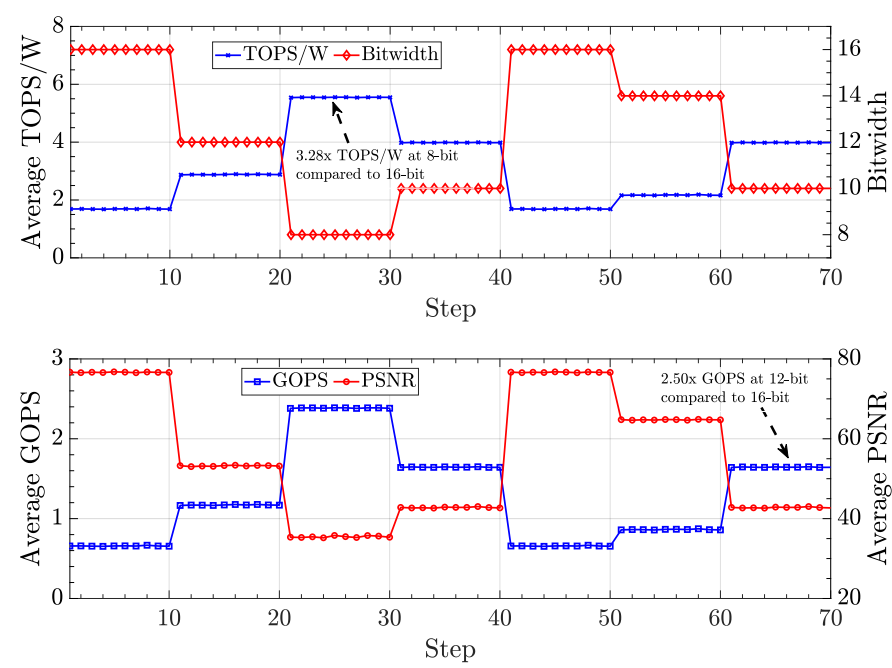

Fig. 15: The performance (GOPS), power (GOPS/W), and quality metrics (PSNR) of IMCA during run time when dynamic approximate computing is enabled
Figure 14 shows the average GOPS of a single IMCA stage with respect to the variable bit widths and number of rows. In 16-bit precision, a single CAM row can achieve 2.5 MOPS. Such a row consists of 64 CAM cells together with the corresponding peripherals (i.e., matching circuit and sense amplifier) and occupies an area less than $100 \mu \mathrm{m}^{2}$. A MAC unit inside the IMCA (i.e., a single CAM row) is $12.83 \mathrm{x}$ more area efficient than traditional arithmetic MAC unit in terms of transistor count. Therefore, a high operational density is provided by in-memory processing. Since the operational complexity in APs depends on the number of bits, not the number of vectors, average GOPS increases as the IMCA size increases as long as the vector fits inside the memory. However, the latency of IMCA is high compared to GPUs and CPUs since a bitwise MAC operation requires many cycles. In addition to providing a superior GOPS rate, the IMCA architecture naturally provides the approximate dynamic computing as introduced in Section III-D. Figure 15 shows results of the IMCA over 7000 convolution operations on $256 \times 256$ images where the bitwidth of the operations are changed dynamically during runtime after every 1000 convolutions. Each step in the figure shows the averaged results for every 100 convolution operations; therefore, there is a total of 70 steps. The bit widths of the processor are set as 16, 12, 8, 10, 16, 14, 10 bits sequentially and change at every 10 steps (i.e., 1000 convolutions). Figure 15 shows the change of bitwidth and corresponding TOPS/W metric of the processor. The figure shows the peak signal to noise ratio (PSNR) and GOPS metrics also for the same corresponding bitwidth.

Figure 16 shows the energy efficiency comparison of various CNN accelerator architectures [40], [53], [51], [24], [54], [55], [56], [57], including the IMCA. For a fair comparison, the IMCA is compared against the architectures, which perform operations over 16-bit fixed-point data with the reported maximum efficiency results. According to the results, the IMCA shows the best energy efficiency, which is $15.49 \%$ better than the state of the art [24]. On the other hand, there are other architectures implemented in more advanced technology and exploiting different techniques such as sparse, analog, or timedomain computing. The sparse architectures are specialized to get benefit from the sparsity of data caused due to the zero weights and activation functions. For example, the SNAP accelerator in [58] performs 1.67 TOPS/W at 16-bit precision and $16 \mathrm{~nm}$ technology for dense sparsity. It shows a significant advantage in sparse networks performing 21.55 TOPS/W at $10 \%$ density benchmarks. In the time-domain approach [59], the data is represented in a time-domain signal with variable pulses. The architecture performs well for fewer bitwidths, achieving $12.08 \mathrm{TOPS} / \mathrm{W}$ on 4-bit inputs and 1-bit weights at $40 \mathrm{~nm}$ technology. In [60], a near-memory computing approach is employed through the inductive coupling between SRAM and processor. The architecture achieves 0.88 TOPS/W at 4-bit precision and $40 \mathrm{~nm}$ technology.

As stated in the introduction section, the two bottlenecks which AI systems struggle with are memory bottleneck and computational complexity since billions of MAC operations are performed on activations iteratively, and each MAC requires at least three memory accesses in the worst case. 


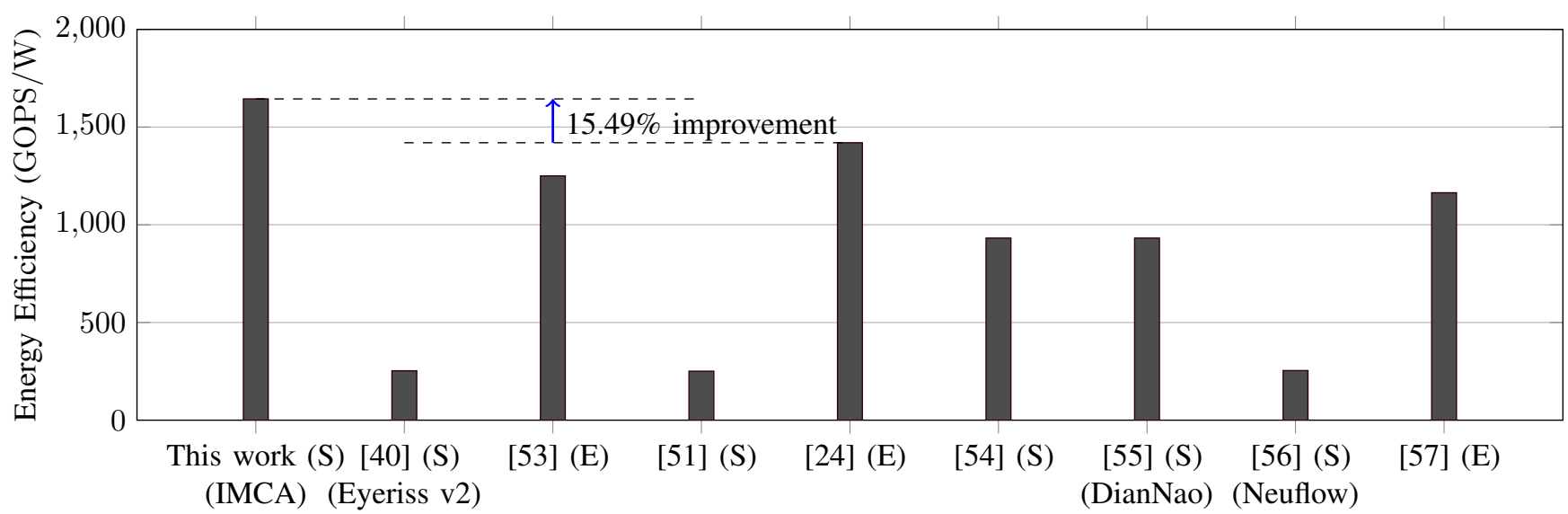

Fig. 16: Energy efficiency (GOPS/W) comparison of convolution accelerators, where S and E indicate the simulation-based and experimentation-based results respectively

The IMCA alleviates these two problems by moving the convolution operation directly inside the memory as well as performing the operations on very dense memory structures. The SRAM memory architectures are also resilient to work on low supply voltages [61] and the IMCA benefit from this opportunity by using a $0.5 \mathrm{~V}$ supply voltage for the CAM cells. The architecture also optimizes the input activation flow between the stages; thus, in-chip bandwidth is maximized. IMCA loads the weigh once for every 2D activation. On the other hand, other accelerator architectures require weight loading more frequently. Also, the weights are not broadcasted through the processing elements, which are the CAM rows in here. A single weight stored in the controller can be reused for MAC operations through a 2D image. The proposed accelerator minimizes the size of the processing element to 64bit CAM cells. Considering the traditional implementation of MAC operation (Figure 3d), which requires at least a $4 \times 16$ bit register as well as the arithmetic logic, IMCA uses only 4x16-bit CAM cells to perform the storage together with the computation. A single CAM cell area approximately equals to single register area, i.e., both 12 transistors. Furthermore, the constant multiplier unit of the IMCA provides a significant advantage over other traditional architectures. It simply skips the logic-0 components of the kernel weights, thus provides an advantage both in performance and power. It also takes the sparsity of kernel weights into account by simply skipping it if it is zero. Considering the statistics of kernel weights of $18 \mathrm{CNN}$ models (Figure 7), $41.4 \%$ of the weights is zero for 8-bit fixed-point representation. This number goes down to $1.3 \%$ for 16 -bit representation. Contrary to other tile-based accelerator architectures, the complexity of the controller stays the same if the number of core increases. The maximum input width of a CNN architecture (NASNet-Large) is 331x331 reported by Google Brain [62]. Another key feature of the IMCA is that contrary to current $\mathrm{CNN}$ architectures that are limited to small input size images $(331 \times 331$ in NASNet Large [52]), IMCA alleviates this issue and allows for larger size images. Considering the reported area results of the fabricated SRAM memories in $65 \mathrm{~nm}$ [63], the proposed single IMCA unit occupies an area less than $0.25 \mathrm{~mm}^{2}$ for CAM depth of 331 and a maximum filter size of $3 \times 3$ (i.e., pipelined CAM memories with a total size of around $23.3 \mathrm{~KB}$ ). At that vector size, an IMCA stage running at 8-bit precision can support around $2.50 \mathrm{GOPS}$ at $0.54 \mathrm{~mW}$ (i.e., $4.63 \mathrm{TOPS} / \mathrm{W}$ ) with a power density of $1.95 \mathrm{~W} / \mathrm{cm}^{2}$. The MAC requirements of some popular CNNs like GoogLeNet, ResNet, MobileNet for realtime operations (i.e., 30 frames per second) changes between 9 and 112 GMACs/s [64]. For a die size around $16 \mathrm{~mm}^{2}$, the IMCA can support a theoretical performance of around 180 GMACs/s. However, a detailed system-wise study is required for a more accurate evaluation.

Compared with Nvidia Tesla P40 [65], whose GPU supports 47 TOPS for INT8 at $250 \mathrm{~W}$ maximum power (i.e., $188 \mathrm{GOPS} / \mathrm{W})$, the proposed architecture can achieve higher power efficiency in terms of GOPS/W (i.e., 4.63 TOPS/W). Even though the GPU on Tesla P40 provides much more general-purpose usage than the proposed in-memory computation architecture, including the hierarchical caches and specific rendering systems, so the comparison is not on the same baseline, the results show the importance of domainspecific architectures [66]. At the same precision, Google TPU (i.e., a domain-specific architecture) achieves 92 TOPS peak throughput performance while consuming $40 \mathrm{~W}$, resulting in the maximum attainable energy efficiency of $2.3 \mathrm{TOPS} / \mathrm{W}$ [67]. Google TPU Edge also reports a theoretical performance of 4 TOPS at $2 \mathrm{~W}$ power consumption (i.e., 2 TOPS/W) at the 8-bit integer precision [68]. Another domain-specific accelerator from Intel, Movidius Myriad 2, can achieve a theoretical performance of 1 TOPS/W efficiency and supports integer operations between 8-64 bits [69].

\section{CONClusion}

In this study, an in-memory convolution engine (IMCA) is proposed, which is based on associative in-memory processing. The proposed accelerator achieves around 1.64 TOPS/W average efficiency at 16-bit fixed-point accuracy, which makes it superior compared with other state of the art accelerators. The architecture supports the fine-grain sparsity-aware computation for more performance and energy gains. Furthermore, 
the architecture provides dynamic approximate computing inherently, a preferred feature for commercial AI accelerators since accuracy requirement changes by $\mathrm{CNN}$ architecture and even the depth of layers within the same architecture. Future work aims to provide an accelerator solution at the system level by integrating the IMCA into a RISC-V based computational system and including solutions for other computational layers such as pooling and normalization layer.

\section{ACKNOWLEDGMENT}

We acknowledge the financial support from AI Initiative, King Abdullah University of Science and Technology (KAUST), Saudi Arabia.

\section{REFERENCES}

[1] D. A. Drachman, "Do we have brain to spare?" Neurology, vol. 64, no. 12, pp. 2004-2005, 2005. [Online]. Available: https: //n.neurology.org/content/64/12/2004

[2] H. Miyazaki et al., "K computer: 8.162 petaflops massively parallel scalar supercomputer built with over 548k cores," in 2012 IEEE International Solid-State Circuits Conference, Feb 2012, pp. 192-194.

[3] F. Jabr, "Does thinking really hard burn more calories?" scientific american, vol. 18, 2012.

[4] V. Sze et al., "Efficient processing of deep neural networks: A tutorial and survey," Proceedings of the IEEE, vol. 105, no. 12, pp. 2295-2329, Dec 2017.

[5] K. He et al., "Delving deep into rectifiers: Surpassing human-level performance on imagenet classification," in 2015 IEEE International Conference on Computer Vision (ICCV), Dec 2015, pp. 1026-1034.

[6] D. Ardila et al., "End-to-end lung cancer screening with threedimensional deep learning on low-dose chest computed tomography," Nature medicine, vol. 25, no. 6, p. 954, 2019.

[7] H. Esmaeilzadeh et al., "Dark silicon and the end of multicore scaling," in 2011 38th Annual International Symposium on Computer Architecture (ISCA), June 2011, pp. 365-376.

[8] A. Coates et al., "Deep learning with cots hpc systems," in Proceedings of the 30th International Conference on International Conference on Machine Learning - Volume 28, ser. ICML'13. JMLR.org, 2013, pp. III-1337-III-1345. [Online]. Available: http: //dl.acm.org/citation.cfm?id=3042817.3043086

[9] N. Jouppi et al., "Motivation for and evaluation of the first tensor processing unit," IEEE Micro, vol. 38, no. 3, pp. 10-19, May 2018.

[10] M. H. Ionica et al., "The movidius myriad architecture's potential for scientific computing," IEEE Micro, vol. 35, no. 1, pp. 6-14, Jan 2015.

[11] M. Horowitz, "1.1 computing's energy problem (and what we can do about it)," in 2014 IEEE International Solid-State Circuits Conference Digest of Technical Papers (ISSCC), Feb 2014, pp. 10-14.

[12] C. C. Foster, Content Addressable Parallel Processors. New York, NY USA: John Wiley \& Sons, Inc., 1976.

[13] H. E. Yantır et al., "A hybrid approximate computing approach for associative in-memory processors," IEEE Trans. Emerg. Sel. Topics Circuits Syst., vol. 8, no. 4, pp. 758-769, Dec 2018.

[14] H. E. Yantir, "Efficient acceleration of computation using associative in-memory processing," Ph.D. dissertation, University of California, Irvine, USA, 2018. [Online]. Available: http://www.escholarship.org/uc/ item/3939f $48 \mathrm{~b}$

[15] H. E. Yantir et al., "An ultra-area-efficient 1024-point in-memory fft processor," Micromachines, vol. 10, no. 8, 2019. [Online]. Available: https://www.mdpi.com/2072-666X/10/8/509

[16] R. Kaplan et al., "A resistive cam processing-in-storage architecture for dna sequence alignment," IEEE Micro, vol. 37, no. 4, pp. 20-28, 2017.

[17] H. E. Yantır et al., "Efficient acceleration of stencil applications through in-memory computing," Micromachines, vol. 11, no. 6, p. 622, Jun 2020. [Online]. Available: http://dx.doi.org/10.3390/mi11060622

[18] L. Yavits et al., "Sparse matrix multiplication on an associative processor," IEEE Trans. Parallel Distrib. Syst., vol. 26, no. 11, pp. 3175-3183, 2015.

[19] M. A. Neggaz et al., "Rapid in-memory matrix multiplication using associative processor," in 2018 Design, Automation Test in Europe Conference Exhibition (DATE), 2018, pp. 985-990.
[20] E. Nurvitadhi et al., "Accelerating binarized neural networks: Comparison of fpga, cpu, gpu, and asic," in 2016 International Conference on Field-Programmable Technology (FPT), Dec 2016, pp. 77-84.

[21] V. Nair et al., "Rectified linear units improve restricted boltzmann machines," in Proceedings of the 27th International Conference on International Conference on Machine Learning, ser. ICML'10. USA: Omnipress, 2010, pp. 807-814. [Online]. Available: http: //dl.acm.org/citation.cfm?id=3104322.3104425

[22] S. Angizi et al., "Imce: Energy-efficient bit-wise in-memory convolution engine for deep neural network," in 2018 23rd Asia and South Pacific Design Automation Conference (ASP-DAC), Jan 2018, pp. 111-116.

[23] Z. Zhou et al., "Edge intelligence: Paving the last mile of artificial intelligence with edge computing," Proceedings of the IEEE, vol. 107, no. 8, pp. 1738-1762, Aug 2019.

[24] J. Sim et al., "14.6 a 1.42tops/w deep convolutional neural network recognition processor for intelligent ioe systems," in 2016 IEEE International Solid-State Circuits Conference (ISSCC), Jan 2016, pp. 264-265.

[25] S. Park et al., "An energy-efficient and scalable deep learning/inference processor with tetra-parallel mimd architecture for big data applications," IEEE Trans. Biomed. Circuits Syst., vol. 9, no. 6, pp. 838-848, Dec 2015.

[26] V. Gokhale et al., "A 240 g-ops/s mobile coprocessor for deep neural networks," in 2014 IEEE Conference on Computer Vision and Pattern Recognition Workshops, June 2014, pp. 696-701.

[27] Z. Du et al., "Shidiannao: Shifting vision processing closer to the sensor," in 2015 ACM/IEEE 42nd Annual International Symposium on Computer Architecture (ISCA), June 2015, pp. 92-104.

[28] Y. Chen et al., " 14.5 eyeriss: An energy-efficient reconfigurable accelerator for deep convolutional neural networks," in 2016 IEEE International Solid-State Circuits Conference (ISSCC), Jan 2016, pp. 262-263.

[29] Jintao Zhang et al., "A machine-learning classifier implemented in a standard 6t sram array," in 2016 IEEE Symposium on VLSI Circuits (VLSI-Circuits), June 2016, pp. 1-2.

[30] A. Shafiee et al., "Isaac: A convolutional neural network accelerator with in-situ analog arithmetic in crossbars," in 2016 ACM/IEEE 43rd Annual International Symposium on Computer Architecture (ISCA), June 2016, pp. 14-26.

[31] P. Yao et al., "Fully hardware-implemented memristor convolutional neural network," Nature, vol. 577, no. 7792, pp. 641-646, 2020. [Online]. Available: https://doi.org/10.1038/s41586-020-1942-4

[32] M. Hu et al., "Memristor crossbar-based neuromorphic computing system: A case study," IEEE Trans. Neural Netw. Learn. Syst., vol. 25 , no. 10, pp. 1864-1878, Oct 2014.

[33] E. Eleftheriou et al., "Deep learning acceleration based on in-memory computing," IBM Journal of Research and Development, vol. 63, no. 6 , pp. 7:1-7:16, Nov 2019.

[34] L. Yavits et al., "Computer architecture with associative processor replacing last-level cache and simd accelerator," IEEE Trans. Comput., vol. 64, no. 2, pp. 368-381, Feb 2015.

[35] A. Agarwal et al., "Leakage power analysis and reduction for nanoscale circuits," IEEE Micro, vol. 26, no. 2, pp. 68-80, March 2006.

[36] G. Razavipour et al., "Design and analysis of two low-power sram cell structures," IEEE Trans. VLSI Syst., vol. 17, no. 10, pp. 1551-1555, Oct 2009.

[37] H. E. Yantir et al., "Approximate memristive in-memory computing," ACM Trans. Embed. Comput. Syst., vol. 16, no. 5s, pp. 129:1-129:18, Sep. 2017. [Online]. Available: http://doi.acm.org/10.1145/3126526

[38] H. E. Yantır et al., "Power optimization techniques for associative processors," Journal of Systems Architecture, vol. 90, pp. 44 - 53, 2018. [Online]. Available: http://www.sciencedirect.com/science/article/ pii/S1383762118300316

[39] J. D. Ullman et al., "Principles of compiler design," Reading: Addison Wesley, 1977.

[40] Y. Chen et al., "Eyeriss v2: A flexible accelerator for emerging deep neural networks on mobile devices," IEEE Trans. Emerg. Sel. Topics Circuits Syst., vol. 9, no. 2, pp. 292-308, June 2019.

[41] M. Jeon et al., "Analysis of large-scale multi-tenant gpu clusters for dnn training workloads," arXiv preprint arXiv:1901.05758, 2019.

[42] S. Gupta et al., "Deep learning with limited numerical precision," in Proceedings of the 32nd International Conference on International Conference on Machine Learning - Volume 37, ser. ICML'15. JMLR.org, 2015, pp. 1737-1746. [Online]. Available: http://dl.acm.org/ citation.cfm?id=3045118.3045303

[43] D. D. Lin et al., "Fixed point quantization of deep convolutional networks," in Proceedings of the 33rd International Conference on International Conference on Machine Learning - Volume 48, ser. ICML'16. JMLR.org, 2016, pp. 2849-2858. [Online]. Available: http://dl.acm.org/citation.cfm?id=3045390.3045690 
[44] M. Rastegari et al., "Xnor-net: Imagenet classification using binary convolutional neural networks," in Computer Vision - ECCV 2016, B. Leibe et al., Eds. Cham: Springer International Publishing, 2016, pp. $525-542$.

[45] H. Zhang et al., "Efficient fixed/floating-point merged mixed-precision multiply-accumulate unit for deep learning processors," in 2018 IEEE International Symposium on Circuits and Systems (ISCAS), May 2018, pp. $1-5$.

[46] T. P. Morgan, "Nvidia pushes deep learning inference with new pascal gpus," Next Platform, September, 2016.

[47] Q. Guo et al., "A resistive tcam accelerator for data-intensive computing," in Proceedings of the 44th Annual IEEE/ACM International Symposium on Microarchitecture, ser. MICRO-44. New York, NY, USA: ACM, 2011, pp. 339-350.

[48] D. Schinkel et al., "A double-tail latch-type voltage sense amplifier with 18ps setup+hold time," in 2007 IEEE International Solid-State Circuits Conference. Digest of Technical Papers, Feb 2007, pp. 314-605.

[49] N. Muralimanohar et al., "Optimizing nuca organizations and wiring alternatives for large caches with cacti 6.0," in 40th Annиal IEEE/ACM International Symposium on Microarchitecture (MICRO 2007), 2007, pp. 3-14.

[50] T. Suzuki et al., "0.5-v, 150-mhz, bulk-cmos sram with suspended bitline read scheme," in 2010 Proceedings of ESSCIRC, 2010, pp. 354-357.

[51] J. Jo et al., "Energy-efficient convolution architecture based on rescheduled dataflow," IEEE Trans. Circuits Syst. I, vol. 65, no. 12, pp. 41964207, 2018

[52] B. Zoph et al., "Learning transferable architectures for scalable image recognition," in Proceedings of the IEEE conference on computer vision and pattern recognition, 2018, pp. 8697-8710.

[53] H. Valavi et al., "A 64-tile 2.4-mb in-memory-computing cnn accelerator employing charge-domain compute," IEEE J. Solid-State Circuits, vol. 54, no. 6, pp. 1789-1799, June 2019.

[54] T. Chen et al., "A high-throughput neural network accelerator," IEEE Micro, vol. 35, no. 3, pp. 24-32, May 2015.

[55] T. Chen et al., "Diannao: A small-footprint high-throughput accelerator for ubiquitous machine-learning," in Proceedings of the 19th International Conference on Architectural Support for Programming Languages and Operating Systems, ser. ASPLOS '14. New York, NY, USA: Association for Computing Machinery, 2014, p. 269-284. [Online]. Available: https://doi.org/10.1145/2541940.2541967

[56] P. Pham et al., "Neuflow: Dataflow vision processing system-on-a-chip," in 2012 IEEE 55th International Midwest Symposium on Circuits and Systems (MWSCAS), Aug 2012, pp. 1044-1047.

[57] J. Lee et al., "Unpu: A 50.6tops/w unified deep neural network accelerator with 1b-to-16b fully-variable weight bit-precision," in 2018 IEEE International Solid - State Circuits Conference - (ISSCC), 2018, pp. 218-220.

[58] J. Zhang et al., "Snap: A $1.67-21.55$ tops/w sparse neural acceleration processor for unstructured sparse deep neural network inference in $16 \mathrm{~nm}$ cmos," in 2019 Symposium on VLSI Circuits, 2019, pp. C306-C307.

[59] A. Sayal et al., "A 12.08-tops/w all-digital time-domain cnn engine using bi-directional memory delay lines for energy efficient edge computing," IEEE J. Solid-State Circuits, vol. 55, no. 1, pp. 60-75, 2020.

[60] K. Ueyoshi et al., "Quest: Multi-purpose log-quantized dnn inference engine stacked on 96-mb 3-d sram using inductive coupling technology in 40-nm cmos," IEEE J. Solid-State Circuits, vol. 54, no. 1, pp. 186196, 2019 .

[61] B. H. Calhoun et al., "A 256-kb 65-nm sub-threshold sram design for ultra-low-voltage operation," IEEE J. Solid-State Circuits, vol. 42, no. 3, pp. 680-688, March 2007.

[62] B. Zoph et al., "Learning transferable architectures for scalable image recognition," CoRR, vol. abs/1707.07012, 2017. [Online]. Available: http://arxiv.org/abs/1707.07012

[63] K. Zhang et al., "Sram design on 65-nm cmos technology with dynamic sleep transistor for leakage reduction," IEEE J. Solid-State Circuits, vol. 40, no. 4, pp. 895-901, April 2005.

[64] V. Sze et al., "Efficient processing of deep neural networks," Synthesis Lectures on Computer Architecture, vol. 15, no. 2, pp. 1-341, 2020.

[65] R. Xu et al., "Deep learning inference on p40 gpus," BIOS, vol. 2, p. 3.

66] D. Patterson, "50 years of computer architecture: From the mainframe cpu to the domain-specific tpu and the open risc-v instruction set," in 2018 IEEE International Solid - State Circuits Conference - (ISSCC), 2018, pp. 27-31.

[67] N. P. Jouppi et al., "In-datacenter performance analysis of a tensor processing unit," in 2017 ACM/IEEE 44th Annual International Symposium on Computer Architecture (ISCA), 2017, pp. 1-12.
[68] Google, "Edge tpu performance benchmarks," data accessed: 11/19/2020. [Online]. Available: https://coral.ai/docs/edgetpu/ benchmarks/

[69] L. A. Libutti et al., "Benchmarking performance and power of usb accelerators for inference with mlperf," in 2nd Workshop on Accelerated Machine Learning (AccML), Valencia, Spain, 2020.

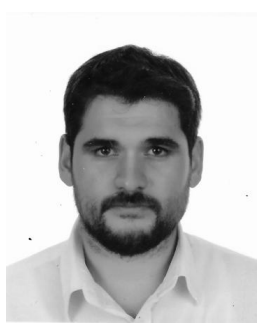

Hasan Erdem Yantir (S'13) received the B.Sc. degree from the computer engineering and electrical and electronics engineering, Yeditepe University, Istanbul, Turkey, in 2011, the M.Sc. degree in computer engineering from Boğaziçi University, Istanbul, and the Ph.D. degree from the University of California, Irvine, CA, USA, in 2018. He is currently a postdoctoral fellow at King Abdullah University of Science and Technology (KASUT), Thuwal, Saudi Arabia. He was an Engineer with Ericsson, Istanbul, from 2011 to 2012, and with Ford Motor Company, Istanbul, from 2012 to 2013. His current research interests include AI accelerator architectures, digital IC design, in-memory computing, computer architectures, and reconfigurable computing.

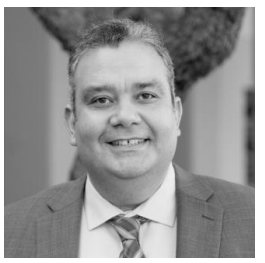

Ahmed M. Eltawil (S'97-M'03-SM'14) received the Doctorate degree from the University of California, Los Angeles, in 2003 and the M.Sc. and B.Sc. degrees (with honors) from Cairo University, Giza, Egypt, in 1999 and 1997, respectively. Since 2019 he is a Professor at the Computer, Electrical and Mathematical Science and Engineering Division (CEMSE) at the King Abdullah University of Science and Technology (KAUST), Thuwal, KSA Since 2005 he was with the Department of Electrical Engineering and Computer Science, at the University of California, Irvine, where he founded the Wireless Systems and Circuits Laboratory. His research interests are in the general area of low power digital circuit and signal processing architectures with an emphasis on mobile systems. Dr. Eltawil has been on the technical program committees and steering committees for numerous workshops, symposia, and conferences in the areas of low power computing and wireless communication system design. He received several awards, as well as distinguished grants, including the NSF CAREER grant supporting his research in low power systems.

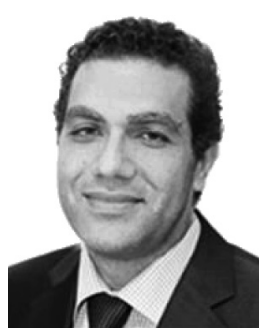

Khaled N. Salama (S'97-M'05-SM'10) received the B.S. degree (Hons.) from the Department of Electronics and Communications, Cairo University, Cairo, Egypt, in 1997, and the M.S. and Ph.D. degrees from the Department of Electrical Engineering, Stanford University, Stanford, CA, USA, in 2000 and 2005, respectively. He was an Assistant Professor with the Rensselaer Polytechnic Institute, Troy, NY, USA, from 2005 to 2009. In 2009, he joined the King Abdullah University of Science and Technology, Saudi Arabia, where was the Founding Program Chair until 2011. He is currently a Professor with the King Abdullah University of Science and Technology. His research on CMOS sensors for molecular detection has been funded by the National Institutes of Health and the Defense Advanced Research Projects Agency. He has authored 225 papers and holds 14 patents on low-power mixed signal circuits for intelligent fully integrated sensors and nonlinear electronics, in particular memristor devices. He was a recipient of the Stanford-Berkeley Innovators Challenge Award in biological sciences. 